\title{
ANNALENDER PHYSIK.
}

JAHRGANG, 818, DRIT'TES STÜCK.

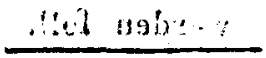

I.

Ueber die Richtiong der Augen;

- d e m.

Direktor VIETH in Dellaü.

Zum vollkommenęa Zuptande des zarten und kunftvollen, Gelichtsorgansas gehört nicht, nue eine fehlerfreie Einrichtfung des Angenbehältoinges felblt und der darịn enthaltenen Hante und Feuchtigkeiten, Condern auch des mechanifchen Apparats; wodurch beide kugellörmige Behälunife bewegt and gorichtet werden. In beiderlei Hinficht, ift das Gelichtsorgan bokannulichn einer Menge von Fehlern unterworfen, welche theils angeboren, theils angewöhnt wegrden; derer nicht zu gedenken, welche von. Kraukheiten und Verletzungen u. dergl. hérrühren: Aneal. d. Puyb4, B, 68. St, 3. J, 2318. St.3. 


\section{[ 234 ]}

Die Felılev.del. Bewegung-and-Richlung-deg Augapfels tind mailtens Sache der. Angewöhnung.

Gehörig gerichlü ilt ein Auge, wenn die Achle deltelben, d. h. die aut die Mille der Hornhant feukrechte Linie, den Punkt trifft, der eigentlich

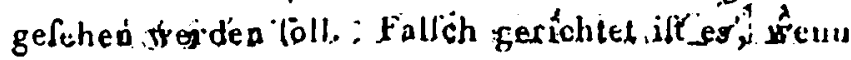
diele Livie den Punkt varbeigeht.

Gehörig gerichtet lind beide Augen, wenn ih.

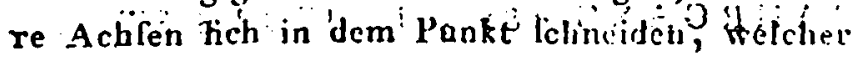
eigentlich gefehen werden foll. lallich gerichtet find lie, wenn diefer Durchlchnittspunkt der beiden Augenoch!en vüter oder entferuter als der rilite Punkt, oder feitswirts defelben fillt.

liei fallcher lichlung def tagen künnan wieder mehtere bulle Stalt finden: nämlich, entwedes ili das eire Auge gehörng gerrchtet, und nur das andere fallch, tiin if gen lind fallich gericltet, -uisd 2 wat entweder beite einwärtg, oder beide auswärtu, oder cins 24 felsr eing-

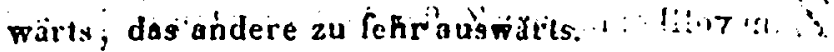

Die Erfolge, welche 'diele perfchit denen' Rielrtungen sur das Sehen haben, Hind reffihiedirn; je nachdem das Auge felhlt fehlerhaft ódei tíchig theIchulfeu ift.

Wenn za. die Achre der Kryltumllinfe nicht in der fenkirecht durch die Mitte der Hornhaut gehenden Hauptachle des Auges' liegt, fo kárn das Auge lich etwas feitwärts richten man̈lten, tum den vificten Punkt gut zu Tehen. Biey nag bei allgebornem Schiolen dertiall feyn. 


\section{$\left[\begin{array}{ll}235 & \text { ] }\end{array}\right.$}

Ilt aber das Ange an fich richlig gebauet, ro wird bei jeder Richtung der Gegenfland, den die Achle des Auges trifti, am deutlichlten, die übrigen feitwärts liegenden aber werden mehroder minder unvollkommen gelehen.

Die Erfolge der verfchiedeneu Richtungen heim Sehen mit beiden. Augen, betreffen die einfache oder doppelte Erfc/leinung der Gegenfiände, und verdienen vorzüglich erürtert zu werdea.

Man tindet in den optifchen Schriften diefes Phänomen auf folgende Art bellimmt, wobei aber, wie es mir lcheint, nicht auf alle U'mltände Kückficht genommen wird: Man fehenämlichdiejenigen Gegenflände einfach, die im Horopter liegen; die andern aber doppelt. Horopter aber loll die Ebene bezeichnen, welche durch den Scheitel des' Winkels, den die Augenachlen bilden, feukrecht auf dellen Ebeue geletzt wird; alfo die Ebenc, die fenkrecht auf das Dreieck $A B P$ Fig. I. durch den Punkt 1 'gelit, deren Durchlchnitt mit der Ebene diefee Dreitcks durch die Linie $S T^{\prime}$ in der Figur angege-: ben ili. Man lelie unter andern den Artikel: "HCropter" in G $\mathrm{G} l$ er's phylikulilichem Wörterbuche. 2. I'. S. 652 .

Ob un diefe Erklärung, oder ob die Erklärung als willkühlich angenommen die Befiimnung des einfachen Sehens richtig fey, müllen wir hiè kürzlich erörtern.

Zurörderlt ilt clas richtig und durch gemeine E. falsung aungemacht, dals der Punkt PFig. 2. auf 


\section{[ 236. ]}

welchen beide Augen gerichtet lind, oder wo dic Augenachlen fich fchneiden, einfach erlcheint. Dieler einzige. Punkt, woraut jedes Mal beide Au-. gen gerichtet lind, und dellen Bilder auf den Netzhüulen gerade cier Pupille gegen über liegen, wird. vollkommen beltiumt und einfach gefehen. Warum? das willen wir vicht. Was wan darüber ge. lugt hat, ill ziemlich gehaltlos.

Nach der obigen Beflimmung nun lollen alle Punkte, dic inr det durch $P$ fenkrecht gefetzten Ebcue liegen, allo z. B. die Punkte $T$ und $S$ ebenfalls vollkommen eisluch gcfehen werden. $-\Lambda$ ber dorüber belelort uns die Érfahrung nicht hialüglich, um es lo ganz heltimmt behaupteu zu können. Man verluche es nur, und hulte die Augen felt auf den Punkt $P, 2$. B. anf die Spitze eines Blcilitits, und halte feitwärts in der Linie $S T$ einen andern Gegenftand, z. B. eine Zirkellpitze, aher ohue die Augeu im mindelten ran dern Punkte $P$ zu verwenden, lo wird nuan wohl nicht iw Stande leyn, beftirnont zu entlcheiden, ol man die Zirkellpitze vollkommen oinfach fehe, und um lo weniger, je weiter fie von der Spitze des Bleiliifts entfernt ilt, weil man fic nicht belitinmt genug lielit.

Nach de robigen Behauplung follen ferner in Gegentheile alle Punkte, : die nicht in der Ebene TS liegen; doppelt erfuheinen. Davon fagt aber die Erlahrung wieder nichts. Man hefte die $A$ u. gen felt auf den Punkt $P, 2$. B. auf die Spitze des dafelbft gehaltenen-Bleiliffts, und halte die Spitzo 


\section{[ $\quad \hat{2} 37$ ]}

aufserhalb TS, etwa in gröfserer Entfernang in $X$ oder näher in $Z$, fo wird man $z$ war wiederum nicht critfcheiden küunen, dafs man fie vollkommen einfach lehe, weil man lie nicht beftimmt genug fieht, abcr viel weniger wird man fagen können, dafs man lie wirklich doppelt lehe; in Gegentheil wird lie, obwohl unbeltimint, einfach erfcheinen.

Auch wirde die Behauptung, dafs nur diejenigen Gegenfiände, die im Horopter, d. h. in der Ebene ST liegen, einfach erlcheinen follen, im $W$ iderfpruche feyn mit der $\Lambda$ ngale des Grundes für die einlache oder doppelte Erlcheinung. Mun lagt nämlich, und das liimmt mit der Erfahrung allerdings überein, das Eintachlehen rühre daher, weil di: Bilder des Gegenliandes auf übereinfiimmende Punkte der Netahaut fallen, und das Doppelfehen vom Gegentheil.

Eigentlich kann man das zwar nicht eine An. gabe des Grundes nennen, es ilt nur eine $\Lambda$ ngabo der Umitände, unter denen die Erlcheinungen erfolgen. Was übcreinftimmende Punkte der Netzhaut lind, wird ebell nicht ganz genau erklürt. Obenhin meint man damit Punkte, die in beiden Augen an einerlei Seile ron den Punkten $A$ und $B$ Yig. 2. liegen, wo die Bilder des Punktes ${ }^{2}$ hinfalIen, auf den die Augenachlen gerichtet find. Aber ob lie gleich weit ron $A$ und $B$, odter ungleich weit von diefen liegen, darüber wird litllichwcigend hiogegangen.

Ob dic fogenannten übereinllimmenden Punkle 


\section{[ 238 ]}

$M$ und $N$, oder beliimmter ausgefprochen: ob diefe Bilder eines Punktes $X$ gleich oder ungleich weil ron $A$ und $B$, den Bildern des Puoktes $P$ liegen, das hängt davon ab, ob die Winkel o und $u$ an der Pupille gleich oder ungleich find. Es ift aber $o=v-x$ und $u=v-p$. In alfo $x$ kleiner als $p$, fo ift $\circ$ gröfser als $u$; ift aber $x$ grölser als $p$, lo ift $o$ kleiner als $u$; und ilt endlich $x$ gleich $p$, fo ilt auch o gleiclsu.

Die Bilder $M$ und $N$ liegen allo in dem Falle gleich weit von $A$ und $B$, wenn die Winkel $p$ und $x$ gleich find, und dielier liall tritt danu cin, wenn $X$ im Umfange eines Kreifes liegt, der durch $O$ und $U$.und $P$ gebt, weil alle Winkel an dielem Kreisumfange diefelbe Sefine $O U$ befpannen.

Verfeht man allo unter dem Ausdrucke ühereinfitmmende Punlie, fulche, die nach einerlei Richtung in beiden Augen, gleichweit von $A$ und $B$ liegen, welches mir die richtige Bedeuluug. $z u$ feyn fcheint, und behauplet sun, man fehe dasjenige einfach, dellen Bilder auf lolche übereinltimmende Punkte fallev, fo lieht man uach diefer Beftim. mung dasjenige einfach, was in der Begränzung einer Spbäre liegt, die durch $U, U, P$ geht, alfo uicht was in der Ebene $S T$ liegt, die man Floropter nenot.

Verlteht man abcr unter übereinflimmende P'enfte nur überhaupt Punkte, die auf einerlei Seite von $A$ und $B$ liegen, fo werden nicht Punkte im Horopter allein, wie etwa $S$, fondern unendlich viele wie $X$ und $Z$ einfach erfcheinen, denn auch 


\section{[ $\begin{array}{lll}239 & \text { ] }\end{array}$}

ron diefen fallen die Bilder an einerlei Seite ron $A$ und $B$.

Jeder Punkt nämlich, welcher aufser/nalb des. parallactifchen Lrinkels $p$ und aufserhalb Jeines Scheilelwintels $q$ liegt, hat feine Bilder in beiden Augen an einerlei Seite von $A$ und $B$, das heilst, beide links odcr beide rechts. Jeder Punkt hingegen, der innerhall des parallactifchen It intels $p$ und innerhalb feines Scheitelwintiels q liegt, hat heine Bilder in beiden Augeu an entgegengefetzten Seiten von $A$ und $B$, das heifst eins liuks, das audere rechts.

Und hiernach kann, wie es mir fcheint, die Beftimmung über das Einfuch - und Doppel - Selaen lo ausgedsückt werden: Was im Scheitel des parallactifchen Winkels felblt liegt, wird beltimmt cinfach gefehen; was innerhalb des parallactifchen Winkels und feines. Scheitelwinkels licgt, wird beflimmt doppelt gefechen, und $z$ war gehen die Erlicheinungen delio weiter aus einander, je weiter der Gegenliand vom Scheitel $\boldsymbol{P}$ entlerut ilt, liey es dielleits oder jenleits les Scheitels; was aulserhalb des parallactilchen Winkels und leines Scheitclwinkels liegt, wird, obwohl unbeltimrol, eirrfach gelehen, wenn es nicbt fehr uahe am Auge ili, in grofser Nälie aber anch unheltimnt doppelt, z. B. der Puukt Y Fig. 1., dellea Bild im linken Ange viel näher an $A$ liegt als in rechten an $B$. Mlan wird dies durch Verluche leicht beliätigt finden.

Welches Bild bei der Doppelerfcheirnung dem 


\section{[ 240. ]}

cinen oder dem andern Auge angehüre, läfst fich durch leichte Verfuche entlcheiden, und ilt auch fchon ohnehin aus Anlicbt der 3. Figur klar. Ein Punkt $V$ nämlich, der innerhalb des parallactilchen Winkels und zwar näher als der Zielpunkt $P$ 'lieg', snacht im linken Auge ein Bild liuks von $A$ und im rechten Auge ein Bild rechts ron $B$. Es erfcheint allo der Punkt $V$ dem linken Auge rechts, dem rechten Auge links von $P$. Ein Punkt $W$ aber, der innerhalb des Scheitelwinkels liegt, macht im lin. ken $A$ uge ein Bild rechts ron $A$, und im rechten Auge ein Bild links ron $B$. Es erlcheint alfo der Punkt $W$ dem linken Auge links, dem recbten Auge rechts rom Zielpunkt $P$.

Wenn allo der doppelt erfcheinende Gegenfiand nälier als der Durchlchnitlspuakt der Augenachfen liegt und man verfchlielist das linke Auge, lo verfichwindet das Bild rechter Hand, rerfchlielst man aber das rechte Auge, fo verfchwindet das Bild linker Fland.

Wern aber der doppelt erfcheinende Gegenliand weiter als der Jurchlichniltspuukt der Augenachleu entfernt ill and man verfchlielst das linke Auge, fo verfchwindet auch das Bild jlinker Hand, und rerlchliefst man das rechte Auge Auge, lo ver. fchwindet das Bild rechter Hand.

Im letztern falle, wo der l'unkt $t \boldsymbol{t}$ jenfeit des Zielpunkts liegt und allo die beiden Bilder delfelben auf der Netzhaut einwärts nach der Nale zu falleu, kann eins dicfer Bilder auch gerade die Stelle 


\section{[ 24: ]}

treffen, wo der Sehenerve in Jdas Augo eintritt; dann verfchwindet diefes Bild von felbft, ohne dafs man das Ange verfchlielst.

Augen, deren äulserer Mechanismus noch unverdorben $i l t$, mülien fich übereinftimmend und willkührlich auf nahe und entfernte Punkte, ouf èin 3 Zoll entferntes Stäubchen und auf einen in unermefslicher Entfernung glänzenden Fixftern richten können. Im erftern Falle fchneiden fich die Augenachlen etwa unter einem Winkel von 60 Grailen, im letztern Falle find fie parallel. Es verdient hierbei hoch angemerkt $2 u$ werden, dars die Augen beim niederwärts gekehrten Blick leicht convergiren, beim aufwärts gekehrten aber lich leichter auf einen entfernten Punkt richten. Daher find Kurzfichtige auch leicht überfichtig; weil es ihnen, wenn lie von unten auf blicken, leichter wird, die :Augen auf den entfernten Punkt zu richten. Weitfichtige find gegentheils für nahe Gegenltäude nieder/ichtig, lie halten z. B. das Buch beim Lefen gern niedrig.

Weiter als parallel können tich gewöhnliche Augen nicht auswärts richten, auch ift eine folcho Richtung, wo die Augenachlen divergiren, die hälslichite die es geben kann. Ganz ohne Beifpiel ift fis jedoch nicht, aber immer als Fehler, nicht als willkührliche 'Thätigkeit der Muskeln.

Hingegen if eine zu fehr convergirende Rich. tung. leider ein lehr oft vorkommender Fehler bei kurzlichtigen Perfonen. Wer unablällig auf ein kaum 6 ' Zoll entferntes Papier blickt, wo er kleino 


\section{[ 242$]$}

Buchltaben oder Figuren mahlt oder betrachlet, difleu inuere Augeumuskelu werderi dadurch endlich fo verkürzl, dafs die Augenachlen nur mit Anftrengung und of gar nicht mehr auf entfernte Punkte, odergar aufunendlich entfernte, das heifst parallel gerichtet werden küınen.

Der Kurzlichlige, der feine Augen bis zú diefem Grad verwahrloft that, enuptindet die Folge lehr unangenehm. Ev lieht die entlernten Gegenliäude nicht nur undeutlich begräuzt wegen der innern Struktur feiner Augen, fonderu, was noch weit Schlinmer ilt, er lieht fie verworren durch einander fchwimmend. An derfelben Stelle, wo dem einen Auge ein entfernter Menfch erlcheint, fieht das andere Auge vielleicht einen entfernten Baum. Das unangenelıme Gefühl, was diefe Verwirrung erregt, belältigt ihn in jeder Gefellichaft, auf jedem Spatziergange. Er findet lich in dem Falle eines Schwindlichten oder Betrunkenen, bei denen auch die Augenmufkeln unfähig lind, beide Angen übercinllimmend auf beftimmte näliere oder entferntere Punkte zu richten und feltzuhalten. Er fieht lich genöthigt, um eiuen entlegenen Gegenltand, wenn auch nicht deutlich begränzt, docli wenigltens einfach und unverworren mit andern zu fehen, das eine Auge zu verfchliefsen. Spricht er mit Jemanden, der ihm nicht ganz nahe fteht, fo fchlïgt er lieber die Augen nieder, weil er den Uebelftand, den die falfche und unlichere Riahtung der Augen hervosbringt, vermeiden will. Wer aus diefem Nie- 


\section{[ 243 ]}

derfchlagen der Augen auf eix böles Gewillon fchliefsen wollte, würde lich eine grofse Unbilligkeit zu Schulden kommen laflen, wie das wohl manchen Phyfiognomen begegnen mag.

Vermuthlich werden Manche, die fo glücklich find, diele Unannehmlichkeilen nicht zu kennen, das Obige für Uebertreibung halten, aber ich weifs, dals viele Kurzlichtige es nur zu fehr durch ihre Erfahrungen beltätigt findea werden.

Uebrigens giebt es auch Kurzlichtige, die ungeachtet der falfchen Richtung der Augenacbfen, die man ihnen anfieht, die Doppelerfcheinung entfernter Gegenfände nicht empfinden, weil he entweder Augen von ungleicher Befchallenheit haben, oder weil fie fich gewöhnt haben, nur mit einem Auge zu fehen. Diefes auf den Gegenftand gerichtete Auge empfängt das Bild auf der Mitte der Netze haut, wo es am lebhafteften und beftimmtelten em. pfunden wird; das andere einwärts gekehrte Auge àber empfängt, feiner Stellung wegen, das Bild weit von der Mitte der Netzhaut einwärts nach der Nale zu, wo es weniger lebhaft empfunden wird, oder, wenn es auf die Stelle trifft, wo der Sehnerve eintritt, ganz verfchwindet. Jenes Bild übertänt diefes letztere, fo wie wenn man vor das eine Auge ein Hohlglas hält, nur diefes deutlichere Bild empfunden wird.

In diefem Falle brauchen fie ent weder immer eins und ebendaffelbe Auge für die entferntern Gegenfiände, oder nach Belinden das eine oder das ande- 


\section{[ 245$]$}

fe. Fällt z. B. in das eine Auge mehr Licht als in das andere, fo ilt gewöhnlich das mehr berchaltete tha. tig, weil das Bild des Gegeuftandes auf defien dunklerm Grunde lebhafter empfunden wird. Es giebt vermuthlich riele, die zeitlebens nur immer mit einem Auge lelien, ohne es zu willen, und das andere gänzliçh in Ruheltand gefetzt haben.

Wenn einmal durchaus gefchielt worden muls, Co if freilich das einfache Schielen erträglicher, als das doppelte, fowohl für den, der es thut, als fur den, der es anfieht, indeflen ilt beides lo unangenehm, dals der Kurzlichtige, der es fich angewöhnt hat, wo möglich fuchen mufs, es fich wieder abzugewöhnen.

Dazu giebt es denn wohl kein anderes Mittel, als im Allgemeinen öftere Uebung, die Augen auf entfernte Punktezu richten und feltzuhalten. Wir wollen uns hierbei noch etwas verweilen. Vielleicht verdiene ich bei manchen Kurzlichtigen, die mit dem bölen Fehler des Doppelfehens behaftet, und gerade nicht mit optifchen Kenntniflen verfehen find, mir einigen Dank, wenn ich darüber etwas ins Detail gehe.

Zuförderit forge man für gleichförmige Erleuchtung, lo dafs in das eire Auge nicht inehr und glänzendercs Licht fallt als in das andere; fonlt wird immer das mehr belchattete Auge thätig: das andere ruhend feyn.

Ferner telle man lich immer fo, dafs der Zielpunkt beider Augen fichtbar, nicht dem ei- 


\section{[ 245 ]}

nen Auge durch cinen zwifchen licgeuden Gegenliand, verdeckl ilt.

Man lehe ferner nicht zu lange auhallend ant. die fehr nahen Gegenftünde, z. B. auf das Papier leim.Lefen, oder Schreiben, oder Zeichnen, fou. dern blicke oft davon weg auf elwas Eutlernteres.

Die fehr nahen Gegenliänds bei feinen Aubeiten, die oft nur zwilichen 3 und 6 Zoll van der, NaSenwurzel lingen, verderben das Auge, fowohl in, Rücklicht leines innern optifchen Struktur, als in Rücticht . Ceives äufseru mechanifchen Bewegungsa apparats ungernein.

Bei dern Sthen auf entfernte Punkte kann es dem Kurzlichligen, der die Fühigkeit verloren hat, die Augenachfen dariuf zu richten, zu nichts helfen, weun er gleich Aafangs fehr entlernte nehmen wollte. Wellen Augeuachfen immer unter einew Winkel von 3o bis to Grad zulummengehen, der wird umfonft einen lixliern tixireu wolleu, er wird jhu jmoer doppelt tehen. Vielinelir nuts er fich begnügen, erlt nur Punkte, die 1,:2, 3 Fuls:rop. den Augen entleint.find, .. feftzuhalten, fo. dafs er. lie vollkommen tivluch; licht. Wenn er merkt, dals. die beiden Bilder aus einander treton, muls er fuchen, fie gleich wieder zur Deckung zu bringen, und wenn ihm dies nicht. möglich.ili, lieber.den Gegenltand um elwas weniges nüher bringen, abs, den Augen geliatten, fich einwärts zu ziehen und in: den leeren Raum zu fchielen.

Auch folgender. Verfuch.kann als eine Uebung. 


\section{$\left[\begin{array}{lll}2 & 246\end{array}\right]$}

der Augen dieneı, un fie aul einen entfernten Punkt zu richten. Man üffne die Schenkel eines Zirkels aus dem Keiszenge lo' weit, dals die Spitzen falt die Entfervurig der-beiden Pupillen der Augen belpannen und halte ihu, das Gewiude gegen die Stiın gckelirt, fo vor das Gelicht; dals die Spizen etras niedriger liegen als dis Geivinde, lo wird man, wenn die Augenachlen lich fo weit gerichtet haben, dals die Zirkellpitzen genau in ihnen liegen, die Erfcheiuung fehen, welche die 4. Figur mit darftellt, niinfich die beiden Schenkel $M M G$ und $M H F$ abgefondeat, und awilichen ihnen einea bis an den Durchfehiiltspunkt der Augenachlen reicheriden Schenkel HQ. Sobald aber die Augenachfen ftirker convergiren, 2. B. lo dafs fie auf den nahern Punkt 1 ' gerichlet lind, allo die 'Zirkellpilzen aufserhalb der. folben liegen, lo wird jener lange Schenkel lich in zwei lich durchkıeuzende Schenkel verwandela; und swar krcuzen lie lich unter einem kleinen Wiakel und nur mit den Spitzen, wenn die Augen nicht viel nähes als auf $Q$ gerichtet lind, hingegen untev einem grüfsern Winkel und mehr nach der Mitte der Schenkel, wenn die Augen fich aut eineu viel nähern Punkt richten.

Man hat alfo hicran ein gutes Mittel, die Augen nach und nach zu einer weniger convergirenden Richtung zu gewöhnen. Man öllne nünlich anfangs den. Zirkel nicht lo weit, Condern nur lo viel, duls man die Erfcheinung des einfach lich darAellundeu lungen Schenkels vorerli ohue grolue Au- 


\section{[ 217 ]}

fireng̈ung hervorhringe. Non ka:ın maw, "rälrend mau dicfe Erfcheinung hetraclitet: den Zirk:I all: mähligetwas melir üffnen, und die Äugeus nöthï: fun, inmer die ein lach erlicheinend đ̈̈ Sp̈itze zu rer: folgen, indinı lich der Schenkel zum Heilpiel vou $P$ bis $Q$ verlönge.t. Er wird fich inmuter in zwrei durchkireuscute schenkel theitcul wollen, weelched mant aber fu lange als müglich zu rerhiddern fuchen mufs.

Nur in Ritckficht der Richtung -der Augenactro fën ilt diefirr V'erlich als ein Verbeflerungsmittel tor' en'pleluled, niclit in Riickticht-der optifclient Structur des Ariges.

Dafs aber in jener Hinlicht dicles Mittel gat iff; und das Suge noch mehr aut den entlernter Punte hin̈leitct, als das hlofse rixiren oises Clegentlandes; 2. B. einor Schreibleder, dic min allmaililig el woj vom Auge entfernt; rührt dahcr, 'weil die Bildere uer Zirkelfpitzen wegen unverütiderter Nähe det letziern immer von gleicher Deutlichketi blèibents duzegen die Feder, bci der allmähligëtí grübiernt Entfernuirg unleutfirh geféhen wird: '.Dbther wird das.Auge Hüi kar gereilzl die Bilder' deir Zirkelfpitj zen, weurr liweh die sichenkel durchkrcuzen, zur Dek: kung zu bringen, als dic aus einauder tretenden undeutlichen Bilder der Feder.

Die Erfelreinung des langen Schenkits' ift, meines Wiffene, von $S$ mith zufallig entdectit rorden:?

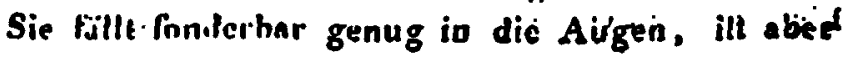
leicht zar entlürea. 


\section{[ $2 ; 3]$}

- Das Bild des linken Sclienkels im rechten Auge briogl die Erfcheinung des abgefonderlen linken Schenkels $M G$ herror, daber auch dieler rerfchwiadet, wena man das rechle Auge verdect t.

Das Bild des rechten Schenkels im linken Augc bringl die Erfcheinung des ah.jelunderten rechteo Schenkels . M// heivor, Jaher auch diefes verIchwindel, wenu man das liake Auge verdeckt.

Die Bilder des recliten Schenkels im rechten und des linken Scheokels im linken Auge fallen, weun die Zirkelfpitzen in den ver!ängerten Augenacliten liegen, aut ubereinliimmeode Stellea der Netzhaule und bringen die Eirfcheinuog des langea Scheokels hervor.

Dic Erfcheioung Fig. 4. entlleht nümlich aus der i.t Fig. 5. dargeflellten; letzlere ili die, liur den liall, wo die Augenachlea aul eiaen naben Punkt gerichtet Gnd, und dio 'Lurke! fpitzen aulserhalb der Yerlängerungell diefer Aclifen falleo. Wie Erfclueinung GNLH gehört desn liaken Auge, dic $g m / 2 \mathrm{dum}$ rechlen Auge un.

i Der Kopf oder das Gerinde des Zirkels, wird vegen feiner Loge nalie an der Stirn nicht gefe: hon, foodern ungefihr our das, was jenfeits der punklirleg Linie XY liegt.

Weon man mit dem Geficht gegen das helle Fenlter gekehit, den Verfuch macht, fo erfclieinen dje fich durclikrcuzenden Schenkel $M / G$ und $m / s$ Fig. 5. den zu convergirend gerichteten Augen, und lo auch der lángè Schenkel MQ Fig. 5. don gelürig 


\section{[ 249 ]}

gerichteten. Augen fichwärzer als die beiden äufsern Schenkel $m_{g}$ und $M H$, woron der Grund nicht. fchwer zu finden ilt.

Nämlich die Erfcheinung $M G$ rührt ber von dem Bilde des linken Schenkels auf der Netzhaut des linken Auges. Von dem linken Schenkel ift aber dem linken Auge die Seite zugekehrt, welche bei jener Slellung ganz im Schatten liegt. So rührt uuch $m h$ her von dem Bilde des rechten Schenkels auf der Netzhaut des rechten Auges; von dem rechten Schenkel ilt aber dem rechten Auge ebenfalls die Schattenleite zugekehrt. Diefe Bilder auf den beiden Netzhüuten der beiden Augen find daher fchwarz, folglich auch der lange Schenkel, der bei gehöriger Richtung der Augen F. 4. erlcheint, weil diefer aus jenen beiden Bildern entlteht, weun fie auf correfpondirende Stellen der beiden Netzhäute fallen. Es ift zum Gelingen des ganzen Verfuchs nöthig, 'dafs beide Zirkellichenkel ron beiden Augen gegen das helle Fenfter oder gegen eine weifse $W$ and gefehen werden. Wenn z. B. dem linken Auge der linke Schenkel vor einer weifsen Wand, dem rechten Auge aber vor einer fchwarzen' $\Gamma$ afel erfcheint, fo gelingt der Verluch nicht gut.

Eine für den Kurzfichtigen nicht unwichtige Frage verdient, zumal jetzt, wo die concaven Brillen fo fehr in der Mode find, hierbei noch erörtert zu werden, nämlich : , lin wie fern können die Hohlو, brillen dazu beitragen, den Febler des DoppelleAuzal. A. Pbyak, B. 53. St. 3. J. 1818. St. 3.

$\mathbf{R}$ 


\section{[ 250$]$}

„hens entfernter Gegenllünde zu vergröfsern oder zu „vermindern," oder welches einerlei ill, "welchen "Einflul's haben die Hohlbrillen auf die Richtung "der Augen?"

Von ihrem Einflufte auf die innero optifche Struktur des Auges ift hier allo nicht dic Redc. Die Hohlbrillen uad lugenanuten Lorgnetten find in diefer Hinlichl für einige ein nothwendiges, für andere ein nicht nothwendiges Uebel, welches defio grölsern Schaden thut, je kleiner die Holbmelrer der Krüınmungen, der hohlen Oberllächen der Glälè, und je kleiner die Zerltreuungsweite der Glasliuleu find, dic man zur Hohlhrille genommen hat.

In Hinficht der Richlung der Augen, häugt, alles übrige gleich geletat, der Einlluls der Brilleu ron der Entlernung der beiden Glafer von tinander ab. Dies zu erläutcrn, mögeu die Figuren 2., 3 , 4. dienen.

Der Ziclpunkt ley in allen dreifiguren der mit $P$ bezcichnete, und $G$ und $F$ feyen die Pankte, wo die von $\boldsymbol{P}$ herkommenden, und durch da: Hohlglas mehr divergirend gewordenen Strahlen lich rerlïngert fchneiden. Alle Strahlen, die ron $P$ auf die Brillenglater fallen, werden to gebrochell, als ob fie ron $G$ und $H$ angingen; $G$ and $H$ lind die beiden geumetrilchen Bilder von $P$.

Die Linien ron $\boldsymbol{P}$ auf die Millen der Glüfer, nämlich $P C^{\prime}$ und $P D$, als die fo gut wie ungebrochen 


\section{$\left[\begin{array}{lll}25 x & 1\end{array}\right.$}

durchgehenden Strahlen wollen wir hier der Kürze wegen die Achfen der Gläfer nennen.

Wenn nun wie in Fig. 2. die beiden Brillengläfer to nahe beifammen ftehen, dals die Achfen $P C$ und $P D$ nicht mehr auf die Pupillen treffen, fo erhalten die Augen Strahlenkegel, deren Achfen $G E$ und $\boldsymbol{G} F$ find, nämlich Strahlen, die ron den nach den äufsern Rändern hin liegenden Stellen des Gla. les, alfo fehr von den Achlen $P C$ und $P D$ divergirend, gebrochen werden. Die geometrifchen Bilder $G$ und $H$ fallen in $A$ und $B$ auf die Mitte der Netzhäute, uud der Punkt $\boldsymbol{P}$ wird einfach gefehen, indem die Augenachfen auf den viel nähern Punkt Q alfo fehr convergirend gerichtet fiud.

Kurzfichtige nun, deren Augen fich durch Verwohnung von felblt auf einen fo nahen Punkt $O$. richten, können allerdings durch eine Handbrille ( Doppellorgnette) deren Gläler fich nach Gefallen einander nähern und von einander entfernẹn la llen, einen entfernten Punkt $\boldsymbol{P}$ ohne Anltrengung einfach fehen, wenn fie die Gläfer nahe zufammenftellen, aber der Fehler der falfchen Richtung und des daraus entitehenden Doppelfehens wird dadurch nur noch mehr befördert und die Handbrille führt darn ihreu undeutfchen Namen mit Recht, der nämlich ein Werkzeug zum Schielen bedeutet.

Wenn aber wie in Fig. 3. die Brillengläler gerade die Entfernung von einander haben, dals die Achfen $P C$ und $P D$ auf die Papillen treffen, alfo mit 


\section{$\left[\begin{array}{ll}252 & 1\end{array}\right.$}

den Augenacblen in einerlei geraden Linien zufamfallen, fo fieht man den Gegenftand $P$, wenn er einfach erfcheint, da wo man ihn mil iblolsen Augen bei gehöriger Richlung lehen würde. Bei etwasentferntenGegenltänden wird derKurzfichlige die einfache Erfcheinung nur durch Anftrengung herbeiführeu können. Der Gegenftand wird ihm bei diefer Stellung derGläfer immer in zwei Bilder aus einander treten wollen. Die Deutlichkeit, die ihm die Hohlgläler gewähren, wird ihm aber jene Anftrengung etwas erleicbtern, und er kann bei einer Handbrille die Entfernung der Gläler von der Fig. 2, his zu der Fig. 3. allmählig vergrö́sern. In fo fern kann allo hier die Handbrille mit beweglichen Gläfern dazu dienen, die Verwöhnung nach und nach zu vermindern.

Wenn endlich wie in Fig. 4. die Brillengläfer fo weit aus einander ftehen, dafs die Augen nur Strahlen von den nach den innern Rändern hin lie. genden Stellen der Gläfer exhalten, fo wird der Punkt $P$ nur einfach gefehen, wenn die Augenach. fen, fo wie die Achlen $G E$ und $H F$ der erhaltenen Sirahlenkegel, in den weiter entfernten Pükt $Q$ zufammengehen.

Wenn allo der Karzfichtige, beim Lefen zum Beifpiel, eine Handbrille von gehöriger nicht zu kleiner Brennweite braucht, fo kann er durch weite Stellung der Gläfer von einander feine Augen nö. thigen, fich nicht auf das in $P$. wirklich befindliche 


\section{[ $\begin{array}{lll}253 & \text { ] }\end{array}$}

Papier, fondern auf eine weiter entfernte Gegend Q zu richten, um die Buchftaben einfach zu Cehen, und dadurch einem ihn bedrohenden Gefichtsfehler entgegen wirken.

Ich glaube, dals diefe Bemerkungen nicht ganz unwichtig für Kurzlichtige find, und es ift mir nicht bekannt, dafs fie fchon fonft, ron Jemand wären mitgetheilt worden.

Ich möchte für die praktilchen Optiker noch einen Vorfchlag hinzufügen, der lehr natürlich aus Obigem folgt. Es ilt folgender: Brillen, die nicht mit der Hand gehalten, fondern aufgefetzt werden, (was freilich bei einem einige Zeit fortdauernden Gebrauche derfelben bequemer ilit), mit folchen Geftellen zu verfehen, woran die Gläfer, etwa durch Schrauben-Vorrichtung, die ein gefshickter Mochaniker leicht dabei anbringen wird, in beliebige|Entfernung foon einader geftellt werden tönnen.

Dellan im December 1817. 